\title{
Geologia
}

\section{Solos Desenvolvidos Sobre Diferentes Rochas Vulcânicas da Ilha de Fernando de Noronha: Padrão de Elementos Terras Raras e Composição Isotópica do Chumbo}

\section{Soils Developed from Different Volcanic Rocks from the Fernando de Noronha Island: Rare-Earth Element Patterns and Isotopic Lead Composition}

\author{
Sonia Maria Barros de O liveiraํㅗ (soniaoli@ usp.br), Luiz Carlos Ruiz Pessenda² (pessenda@ cena.usp.br), \\ Marly Babinski (babinski@ usp.br), Simone Maria Costa Lima Gioia3 (sgioia2010@ gmail.com), \\ Deborah Inês Teixeira Fávaro4 (defavaro@ipen.br) \\ 1Departamento de Mineralogia e Geotectônica - Instituto de Geociências - USP \\ R. do Lago 562, CEP 05508-080, São Paulo, SP, BR \\ ${ }^{2}$ Centro de Energia Nuclear na Agricultura (CENA) - USP, Piracicaba, SP, BR \\ ${ }^{3}$ Centro de Pesquisas Tecnológicas da Marinha, São Paulo, SP, BR \\ ${ }^{4}$ Comissão Nacional de Energia N uclear - Instituto de Pesquisas Energéticas e Nucleares, São Paulo, SP, BR
}

Recebido em 19 de abril de 2011; aceito em 04 de outubro de 2011

\section{RESUMO}

Este estudo focaliza a influência da pedogênese no comportamento dos elementos terras raras em solos derivados de diferentes tipos litológicos que se alteraram sob as mesmas condições de clima tropical úmido. Investiga também a possível contribuição do $\mathrm{Pb}$ aerotransportado a esses solos. Foram estudados 5 perfis de solo desenvolvidos sobre diferentes rochas vulcânicas aflorantes na ilha de Fernando de Noronha. Os resultados mostram que, de modo geral, o intemperismo concentra os ETR, mantendo aproximadamente o mesmo padrão de distribuição da rocha parental, com ligeiro enriquecimento preferencial dos ETRP. Os dados isotópicos de $\mathrm{Pb}$ indicaram a presença de um componente antrópico não radiogênico nos horizontes superiores dos solos.

Palavras-chave: Solos; Fernando de Noronha; Geoquímica; Elementos terra raras; Isótopos de Pb.

\begin{abstract}
This study examines the influence of pedogenesis on the distribution of rare earth elements in soils derived from different rock types and formed under tropical humid climate, as well as the possible contribution of airborne $\mathrm{Pb}$ to these soils. We studied 5 soil profiles developed from different volcanic rocks cropping out in the Fernando de Noronha island. Results show that in the course of weathering, the soils were enriched in REE. The REE patterns of the soils are similar to those of the parent material, except for a slight HREE enrichment. Lead-isotope data indicate the presence of a nonradiogenic anthropogenic component in the upper horizons of the soil profiles.
\end{abstract}

Keywords: Soils; Fernando de Noronha island; Geochemistry; REE; Pb isotopes. 


\section{INTRODUÇÃO}

$\mathrm{O}$ arquipélago de Fernando de Noronha situa-se no oceano Atlântico, a latitude de $3^{\circ} 51^{\prime} \mathrm{S}$ e $32^{\circ} 25^{\prime} \mathrm{W}$, a cerca de $400 \mathrm{~km}$ da costa brasileira. Mais de $90 \%$ da área total do arquipélago concentra-se na ilha do mesmo nome que, embora com apenas cerca de $17 \mathrm{~km}^{2}$, apresenta uma razoável variedade litológica. Nela afloram rochas vulcânicas pertencentes basicamente a duas formações, que foram descritas no trabalho pioneiro de Almeida (1955). A mais antiga, Formação Remédios, é composta por plugs e domos de fonólitos, traquitos e álcali-basaltos, cortados por diques basaníticos e lamprofíricos. Sobreposta a essas rochas está a Formação Quixaba, constituída essencialmente por lavas de nefelina basaltos. A ilha apresenta relevo de planaltos suavemente ondulados, com altitudes que alcançam até $320 \mathrm{~m}$, e vertentes rochosas abruptas. Os solos mais desenvolvidos situam-se no planalto sobre os basaltos da Formação Quixaba, e foram classificados como cambissolos (Marques, 2004). A precipitação média anual é de $1.300 \mathrm{~mm}$, concentrada na estação úmida que vai de março a maio. As temperaturas variam pouco durante o ano, com uma média situando-se em torno de $25^{\circ} \mathrm{C}$.

O objetivo deste trabalho foi o de investigar o comportamento dos elementos terra raras (ETR) nos diferentes tipos de solos, derivados de diferentes litologias, que evoluíram sob as mesmas condições de clima tropical úmido. Apesar de suas baixas solubilidades, os ETR podem ser mobilizados durante o intemperismo, e sofrer fracionamento. No entanto, a migração dos ETR geralmente não se dá a longas distâncias, de modo que esses elementos acabam reciclados dentro dos próprios perfis dos solos, sendo redistribuídos entre os horizontes. Assim solos e regolitos constituem-se em reservatórios de longo prazo para a acumulação de ETR, e podem mesmo constituir concentrações de interesse econômico, quando a rocha parental é favorável (Oliveira e Imbernon, 1998).

Por outro lado, por estar Fernando de Noronha situado em região remota, distante das fontes diretas de poluição industrial, seus solos constituem-se em material especialmente adequado para a investigação do aporte de poluentes aerotransportados. No presente trabalho será também investigada a contribuição do $\mathrm{Pb}$ atmosférico aos solos por meio do estudo de sua composição isotópica.

\section{MATERIAIS E MÉTODOS}

Foram amostrados 5 perfis de solo desenvolvidos sobre diferentes tipos de rochas vulcânicas, nas seguintes localidades, mostradas no mapa da Figura 1: Morro do Quixaba (MQ), Baía do Sancho (SB), Mata Ponta Oeste (MP), Ponta de Atalaia (AB) e Praia do Leão (LB). As localidades
MQ, SB e MP situam-se no planalto formado pelos nefelina basaltos da Formação Quixaba, as altitudes de 170, 65 e $100 \mathrm{~m}$, respectivamente. Os perfis de solo nessas localidades são bem desenvolvidos e classificados como cambissolos (Marques, 2004). As localidades LB e AB situam-se ao nível do mar, sendo que em LB o perfil está no contato entre os nefelina basaltos da Formação Quixaba e um pequeno domo de traquito alcalino da Formação Remédios. Em AB, o perfil desenvolveu-se sobre um "sill" de essexito da Formação Remédios, cortado por um dique máfico. Tanto em LB com em AB o perfil de solo é raso e pouco evoluído.

Em MQ foi aberta uma trincheira e o perfil foi amostrado a cada $10 \mathrm{~cm}$ até $290 \mathrm{~cm}$. Nas demais localidades, os perfis foram amostrados por tradagem, a cada $10 \mathrm{~cm}$; a profundidade de amostragem atingiu $140 \mathrm{~cm}$ em SB, $120 \mathrm{~cm}$ em MP, $70 \mathrm{~cm}$ em AB e $40 \mathrm{~cm}$ em LB. Um estudo prévio (Oliveira et al., submetido) tratou da caracterização mineralógica e geoquímica desses solos e de seus conteúdos em alguns metais (Co, $\mathrm{Cu}, \mathrm{Cr}, \mathrm{Ni}, \mathrm{Zn}$ e $\mathrm{Hg}$ ). Para o presente trabalho, 49 amostras foram analisadas para os seguintes elementos terras raras: $\mathrm{La}, \mathrm{Ce}, \mathrm{Nd}, \mathrm{Sm}, \mathrm{Eu}, \mathrm{Tb}, \mathrm{Yb}, \mathrm{Lu}$. Para os fins de determinação das razões isotópicas de $\mathrm{Pb}$, foram utilizadas amostras do perfil MQ às profundidades de 0 - 10, 20 - 30, 40 - 50 e 60 - $70 \mathrm{~cm}$. Para os demais perfis (MP, SB, AB e LB), foram utilizadas amostras coletadas às profundidades de $0-10$ e $10-20 \mathrm{~cm}$.

A determinação dos teores dos elementos terras raras foi realizada pela técnica de ativação com nêutrons (NAA). As amostras, materiais de referência e padrões sintéticos foram irradiados por $16 \mathrm{~h}$, sob um fluxo de nêutrons térmi$\cos$ de $10^{12} \mathrm{n} \mathrm{cm}^{-2} \mathrm{~s}^{-1}$ no reator nuclear de pesquisa IEA-R1,

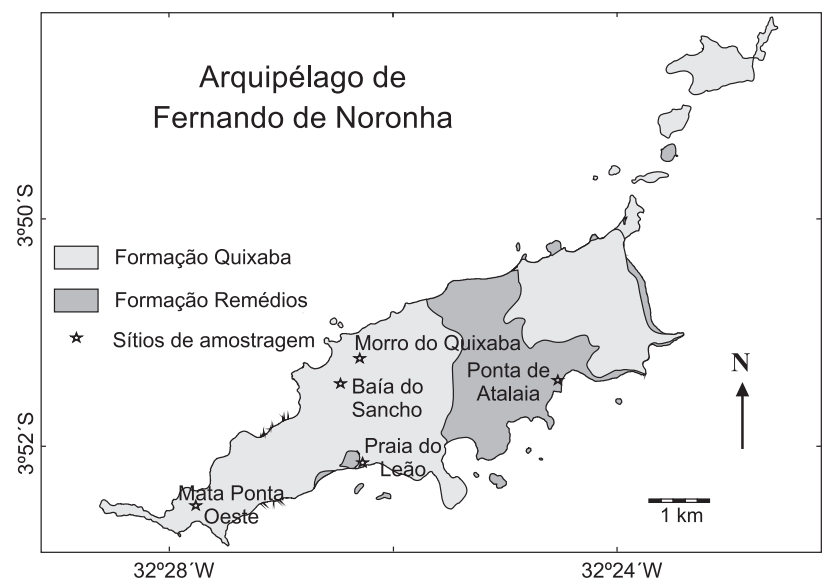

Figura 1. Mapa geológico do Arquipélago de Fernando de Noronha (simplificado de Almeida, 1955), e localização dos pontos de amostragem dos perfis de solo. 
no IPEN São Paulo. A validação do método da NAA, em termos de precisão e exatidão, foi realizada por meio da análise dos materiais de referência Buffalo River Sediment (NIST RM 8704), Estuarine Sediment (NIST SRM 1646a) e BEN (Basalt-IWG-GIT). Os valores (ppm) de LD (limite de detecção) e LQ (limite de quantificação), respectivamente, para os ETR foram os seguintes: La 0,07 e 0,20; Ce 0,19 e 0,57; Nd 0,49 e 1,46; Sm 0,01 e 0,04; Eu 0,009 e 0,027; Tb 0,07 e 0,20; Yb 0,03 e 0,10; Lu 0,003 e 0,009.

As razões isotópicas de chumbo foram determinadas 1. na amostra total, o que envolveu dissolução total; 2. no lixiviado, o que mostraria possível componente antrópico, se este existir, já que esse chumbo é facilmente liberado com ácidos fracos; e 3. no resíduo, que contém chumbo dos retículos cristalinos dos minerais, conhecido como chumbo geogênico. Para a dissolução total, as amostras (100 mg) foram dissolvidas com $\mathrm{HF}+\mathrm{HNO}_{3}+\mathrm{HCl}(3: 1: 6)$ em bombas PARR. Os lixiviados foram obtidos com uma mistura de $\mathrm{HNO}_{3} 1 \mathrm{~N}$ e $\mathrm{HCl} 1 \mathrm{~N}$ reagindo com os solos por $4 \mathrm{~h}$ numa placa aquecida a $60^{\circ} \mathrm{C}$, segundo os procedimentos expostos no trabalho de Gioia (2004). As razões isotópicas de $\mathrm{Pb}$ foram determinadas usando um espectrômetro de massa multicoletor VG 354. O material de referência utilizado foi Columbia River Basalt, USGS, BCR-1 (Woodhead e Hergt, 2000) que forneceu uma concentração de $\mathrm{Pb}$ de 13,127 \pm $0,27 \mathrm{ppm}$, e as seguintes razões isotópicas: ${ }^{206} \mathrm{~Pb} /{ }^{204} \mathrm{~Pb}=$ $18,745 \pm 0,012 ;{ }^{207} \mathrm{~Pb} /{ }^{204} \mathrm{~Pb}=15,631 \pm 0,029 ;{ }^{208} \mathrm{~Pb} /{ }^{204} \mathrm{~Pb}=$ $38,653 \pm 0,090$. As análises foram realizadas no Centro de Geocronologia da Universidade de São Paulo.

\section{RESULTADOS E DISCUSSÃO}

\section{Elementos terras raras}

Os teores dos ETR nas amostras de solos estão listados na Tabela 1, juntamente com os cálculos de $\Sigma$ ETR $(\mathrm{La}+\mathrm{Ce}+\mathrm{Nd}+\mathrm{Sm}+\mathrm{Eu}+\mathrm{Tb}+\mathrm{Yb}+\mathrm{Lu})$ e da razão $(\mathrm{La} / \mathrm{Yb})_{\mathrm{N}}$, que quantifica o grau de fracionamento entre os terras raras leves ETRL $(\mathrm{La}+\mathrm{Ce}+\mathrm{Nd}+\mathrm{Sm})$ e os terras raras pesadas $\operatorname{ETRP}(\mathrm{Eu}+\mathrm{Tb}+\mathrm{Yb}+\mathrm{Lu})$. Adicionalmente foram mostrados os valores das razões $\mathrm{Si} / \mathrm{Al}$, que são um indicador do grau de alteração do material, uma vez que o intemperismo provoca lixiviação da sílica e manutenção do Al. Constam também da tabela os teores de ETR para as rochas que constituem o material parental dos solos estudados (Weaver, 1990; Lopes, 2002), e a média da crosta continental superior (Wedepohl, 1995). A Figura 2 apresenta os padrões dos elementos terras raras normalizados em relação ao condrito para os solos estudados e para o material parental.

Em geral, os solos de Fernando de Noronha são ricos em ETR, com valores bastante superiores aos das médias crustais (Tabela 1) e aos de solos comumente desenvolvidos sobre rochas graníticas (Weijden e Weijden, 1995; Minarik et al., 1998; Caspari et al., 2006). Isso se deve ao fato de o material parental ser naturalmente enriquecido nesses elementos em relação às rochas crustais mais comuns. Além disso, de modo geral, a pedogênese em ambiente tropical úmido provoca a intensa mobilização dos elementos mais solúveis, tais como os alcalinos e alcalino terrosos, e parte da sílica, concentrando relativamente os mais insolúveis, como é o caso dos ETR.

Considerando os solos desenvolvidos sobre os nefelina basaltos da Formação Quixaba, a ETR varia de 230 a 695 ppm no perfil MQ, de 298 a 482 ppm no perfil SB, e de 465 a 564 ppm no perfil MP. Esses valores são superiores aos encontrados nos nefelina basaltos frescos, onde a $\Sigma E T R$ varia de 253 a 311 ppm (Lopes, 2002; Weaver, 1990), o que indica que os ETR concentram-se durante a formação dos solos. Nos perfis MQ e SB, onde há uma variação considerável da razão $\mathrm{Si} / \mathrm{Al}$, há correlação negativa significativa $(\mathrm{p}<0,001)$ entre essa razão e $\Sigma$ ETR $\left(\right.$ em MQ $\mathrm{r}^{2}=0,91$ e em SB $\left.r^{2}=0,74\right)$, mostrando que quanto mais intensa a alteração, maior a concentração de ETR. Na Figura 2, a faixa de variação dos ETR, das amostras do Morro do Quixaba situadas às profundidades de $0-130 \mathrm{~cm}(\mathrm{Si} / \mathrm{Al}$ entre $0,8$ e 0,9$)$, aparece nitidamente acima da faixa de variação das amostras situadas às profundidade de 140-290 cm (Si/ Al entre 1,0 e 1,2). Na Baía do Sancho, o mesmo se repete, com a faixa de variação dos ETR para os solos de profundidades 0 - $110 \mathrm{~cm}(\mathrm{Si} / \mathrm{Al}=1,0)$ situada acima daquela correspondente à dos solos de $110-140 \mathrm{~cm}(\mathrm{Si} / \mathrm{Al}$ entre 1,19 e 1,46). O perfil do Morro do Quixaba mostra um amplo intervalo de variação da razão $(\mathrm{La} / \mathrm{Yb})_{\mathrm{N}}(16,0$ - 40,6), e uma correlação negativa significativa entre $\Sigma$ ETR e (La/ $\mathrm{Yb})_{\mathrm{N}}\left(\mathrm{r}^{2}=-0,83, \mathrm{P}<0,001\right)$, o que indica que quanto mais os ETR se acumulam, maior é a acumulação preferencial de ETRP. Para os solos da Baía do Sancho e da Mata Ponta Oeste, os padrões de ETR subparalelos (Figura 2) e o pequeno intervalo de variação da razão $(\mathrm{La} / \mathrm{Yb})_{\mathrm{N}}$ dentro dos perfis (Tabela 1) não apontam fracionamento significativo durante o intemperismo.

Os perfis desenvolvidos sobre as rochas mais ácidas da Formação Remédios - Ponta de Atalaia e Praia do Leão - são perfis rasos, pouco diferenciados, o que fica evidente pela modesta variação da razão $\mathrm{Si} / \mathrm{Al}$ em cada um deles (AB: 1,85 - 1,90; LB: 1,94 - 1,98). A faixa de variação dos teores de ETR também é limitada nesses perfis (Figura 2). A $\Sigma$ ETR está entre 335 e 407 ppm em AB, e entre 500 a 547 ppm em LB. Não se percebe, tampouco, nesses solos sinais de fracionamento dos ETR, o que é evidenciado pelo estreito intervalo de variação da razão $(\mathrm{La} / \mathrm{Yb})_{\mathrm{N}}$ dentro dos perfis (Tabela 1$)$. A exemplo dos solos derivados das rochas da Formação Quixaba, os solos da Praia do Leão, derivados dos traquitos da Formação Remédios, mostram-se enriquecidos em relação 
Tabela 1. Teores dos elementos terras raras (ppm), razão (La/Yb) e razão Si/Al dos solos da ilha de Fernando de Noronha, dos nefelina basaltos, essexito e traquito (Lopes, 2002; Weaver, 1990), e da média da crosta continental superior (UCC: Wedepohl, 1995).

\begin{tabular}{|c|c|c|c|c|c|c|c|c|c|c|c|}
\hline & La & $\mathrm{Ce}$ & Nd & Sm & Eu & $\mathbf{T b}$ & $\mathrm{Yb}$ & Lu & $(\mathrm{La} / \mathrm{Yb})_{N}$ & $\Sigma E T R$ & Si/Al \\
\hline \multicolumn{12}{|l|}{$M Q \mathrm{~cm}$} \\
\hline $0-10$ & 148 & 322 & 142 & 28,0 & 9,0 & 4,2 & 5,4 & 0,75 & 18,4 & 659 & 0,81 \\
\hline $20-30$ & 146 & 330 & 131 & 30,0 & 9,3 & 3,5 & 6,1 & 0,82 & 16,0 & 657 & 0,80 \\
\hline $40-50$ & 150 & 334 & 123 & 26,0 & 8,1 & 3,2 & 5,3 & 0,60 & 19,0 & 650 & 0,85 \\
\hline $50-60$ & 144 & 287 & 122 & 22,0 & 6,6 & 2,3 & 4,7 & 0,55 & 20,5 & 589 & 0,88 \\
\hline $70-80$ & 169 & 310 & 138 & 27,0 & 7,3 & 3,1 & 5,1 & 0,65 & 22,2 & 660 & 0,89 \\
\hline $100-110$ & 169 & 328 & 157 & 25,0 & 7,4 & 3,0 & 5,1 & 0,63 & 22,2 & 695 & 0,92 \\
\hline $120-130$ & 163 & 288 & 134 & 21,0 & 6,2 & 1,9 & 4,0 & 0,56 & 27,3 & 619 & 0,93 \\
\hline $140-150$ & 132 & 204 & 82 & 13,0 & 3,5 & 1,4 & 2,1 & 0,24 & 42,1 & 438 & 0,99 \\
\hline $160-170$ & 127 & 191 & 61 & 12,0 & 3,5 & 1,6 & 1,8 & 0,15 & 47,3 & 398 & 1,05 \\
\hline $180-190$ & 106 & 150 & 45 & 9,0 & 2,8 & 0,8 & 1,8 & 0,15 & 39,5 & 316 & 1,13 \\
\hline $200-210$ & 117 & 180 & 47 & 10,0 & 3,1 & 0,6 & 1,8 & 0,21 & 43,6 & 360 & 1,36 \\
\hline $220-230$ & 81 & 106 & 32 & 7,0 & 2,0 & 0,5 & 1,6 & 0,16 & 33,9 & 230 & 1,46 \\
\hline $240-250$ & 121 & 184 & 86 & 15,0 & 3,3 & 1,3 & 2,2 & 0,20 & 36,9 & 413 & 1,19 \\
\hline $250-260$ & 110 & 165 & 78 & 15,0 & 3,0 & 0,4 & 2,1 & 0,20 & 35,1 & 374 & 1,30 \\
\hline $270-280$ & 103 & 155 & 91 & 14,0 & 2,8 & 1,0 & 1,7 & 0,20 & 40,6 & 369 & 1,22 \\
\hline $280-290$ & 105 & 158 & 89 & 13,0 & 2,9 & 0,7 & 2,1 & 0,20 & 33,5 & 371 & 1,16 \\
\hline \multicolumn{12}{|l|}{$\mathrm{SB} \mathrm{cm}$} \\
\hline $0-10$ & 101 & 221 & 92 & 21,1 & 6,2 & 2,0 & 3,4 & 0,39 & 19,9 & 447 & 1,02 \\
\hline $10-20$ & 115 & 226 & 102 & 22,7 & 6,9 & 2,5 & 3,8 & 0,46 & 20,3 & 479 & 1,03 \\
\hline $20-30$ & 96 & 213 & 95 & 21,2 & 6,2 & 2,1 & 4,3 & 0,49 & 15,0 & 438 & 1,04 \\
\hline $40-50$ & 83 & 193 & 78 & 16,4 & 5,1 & 1,7 & 3,7 & 0,39 & 15,0 & 381 & 1,03 \\
\hline $60-70$ & 101 & 225 & 97 & 21,2 & 5,7 & 2,3 & 3,6 & 0,41 & 18,8 & 456 & 1,04 \\
\hline $80-90$ & 112 & 237 & 96 & 22,6 & 7,3 & 2,7 & 3,7 & 0,38 & 20,3 & 482 & 1,03 \\
\hline $100-110$ & 94 & 185 & 71 & 15,1 & 4,8 & 1,3 & 3,1 & 0,39 & 20,3 & 375 & 0,99 \\
\hline $110-120$ & 70 & 153 & 53 & 12,8 & 4,1 & 1,7 & 2,7 & 0,40 & 17,4 & 298 & 1,19 \\
\hline $120-130$ & 70 & 159 & 56 & 13,4 & 4,3 & 1,5 & 3,2 & 0,42 & 14,7 & 308 & 1,24 \\
\hline $130-140$ & 70 & 156 & 58 & 11,1 & 4,5 & 1,7 & 3,0 & 0,44 & 15,6 & 305 & 1,46 \\
\hline \multicolumn{12}{|l|}{$M P \mathrm{~cm}$} \\
\hline $0-10$ & 132 & 271 & 124 & 23,1 & 6,7 & 2,8 & 3,3 & 0,64 & 26,8 & 564 & 1,01 \\
\hline $10-20$ & 111 & 248 & 95 & 18,1 & 5,1 & 2,3 & 2,8 & 0,65 & 26,6 & 483 & 0,93 \\
\hline $20-30$ & 123 & 257 & 95 & 19,6 & 5,5 & 1,8 & 2,4 & 0,49 & 34,4 & 505 & 0,93 \\
\hline $40-50$ & 124 & 267 & 112 & 19,8 & 5,8 & 1,9 & 3,1 & 0,67 & 26,8 & 534 & 1,08 \\
\hline $60-70$ & 120 & 261 & 120 & 18,4 & 5,5 & 1,7 & 2,9 & 0,40 & 27,7 & 530 & 1,00 \\
\hline $89-90$ & 108 & 273 & 112 & 17,4 & 5,1 & 2,1 & 3,6 & 0,40 & 20,1 & 522 & 0,94 \\
\hline $100-110$ & 113 & 264 & 99 & 17,1 & 5,0 & 1,7 & 3,5 & 0,38 & 21,6 & 504 & 1,07 \\
\hline $110-120$ & 111 & 240 & 87 & 16,7 & 4,9 & 1,8 & 3,1 & 0,35 & 24,0 & 465 & 1,03 \\
\hline
\end{tabular}


Tabela 1. (continuação)

\begin{tabular}{cccccccccccc}
\hline & La & $\mathbf{C e}$ & $\mathbf{N d}$ & $\mathbf{S m}$ & $\mathbf{E u}$ & $\mathbf{T b}$ & $\mathbf{Y b}$ & $\mathbf{L u}$ & $\mathbf{( L a / Y b})_{\mathbf{N}}$ & $\boldsymbol{\Sigma}$ ETR & Si/Al \\
\hline $\mathrm{AB}$ cm & & & & & & & & & & & \\
$0-10$ & 89 & 183 & 43 & 12,5 & 3,0 & 1,0 & 2,5 & 0,53 & 23,9 & 335 & 1,85 \\
$10-20$ & 95 & 194 & 64 & 13,2 & 3,3 & 1,1 & 2,4 & 0,56 & 26,5 & 374 & 1,85 \\
$40-50$ & 99 & 200 & 57 & 13,9 & 3,4 & 1,2 & 2,4 & 0,39 & 27,6 & 377 & 1,87 \\
$50-60$ & 104 & 212 & 68 & 15,3 & 3,7 & 1,3 & 2,6 & 0,36 & 26,8 & 407 & 1,90 \\
$60-70$ & 98 & 203 & 68 & 14,1 & 3,4 & 1,1 & 2,6 & 0,68 & 25,3 & 391 & 1,85 \\
\hline LB cm & & & & & & & & & & & \\
$0-10$ & 139 & 261 & 118 & 18,4 & 5,3 & 1,7 & 3,1 & 0,50 & 30,1 & 547 & 1,98 \\
$10-20$ & 139 & 260 & 106 & 18,3 & 5,4 & 1,7 & 2,9 & 0,50 & 32,1 & 534 & 1,96 \\
$20-30$ & 138 & 257 & 105 & 18,2 & 5,3 & 1,7 & 3,2 & 0,40 & 28,9 & 529 & 1,94 \\
$30-40$ & 130 & 241 & 102 & 17,2 & 5,0 & 1,7 & 2,7 & 0,40 & 32,3 & 500 & 1,94 \\
\hline nebas & 72 & 154 & 71 & 14,0 & 3,9 & 1,6 & 2,00 & 0,27 & 28,8 & 319 & \\
nebas & 69,5 & 141 & 67 & 12,2 & 3,9 & 1,4 & 1,75 & 0,22 & 26,6 & 296 & \\
nebas & 60,4 & 117 & 59 & 11,0 & 3,2 & 1,2 & 1,56 & 0,16 & 26,0 & 254 & \\
essex & 115 & 167 & 43 & 6,6 & 1,9 & 0,8 & 1,79 & 0,24 & 43,1 & 336 & \\
traq & 121 & 171 & 39 & 4,6 & 1,4 & 0,5 & 2,17 & 0,36 & 37,4 & 339 & \\
traq & 122 & 175 & 38 & 4,5 & 1,3 & 0,5 & 2,20 & 0,36 & 37,4 & 345 & \\
UCC & 32,3 & 65,7 & 25,9 & 4,7 & 1,0 & 0,5 & 1,50 & 0,27 & 3,5 & 132 & \\
\hline
\end{tabular}

ao material parental, que apresenta $\Sigma E T R$ na faixa de 339 a 345 ppm, segundo Lopes (2002) e Weaver (1990). Já os solos da Ponta de Atalaia são pouco enriquecidos em ETR em relação ao material parental, composto de rochas essexíticas, com $\Sigma E T R$ de cerca de 336 ppm (Lopes, 2002).

Em contexto geológico e climático semelhante ao do arquipélago de Fernando de Noronha, a alteração intempérica dos basaltos sujeitos à exfoliação esferoidal no Havaí mostrou que há enriquecimento em ETR nas porções internas menos alteradas, mais próximas aos núcleos de rocha fresca, enquanto que nas bordas mais alteradas dos núcleos há empobrecimento em ETR (Patino et al., 2003). Por outro lado, o intemperismo de anfibolitos, sob clima semiárido, na Índia, levou a expressiva concentração de ETR nos produtos da alteração (Sharma e Rajamani, 2000). Em ambas as situações, tal como nos solos de Fernando de Noronha, foi observado discreto fracionamento dos ETR durante o intemperismo.

\section{Isótopos de chumbo}

A Tabela 2 mostra o conjunto de dados da composição isotópica de $\mathrm{Pb}$ nos solos (amostra total, lixiviado e resíduo). Os intervalos de variação das razões ${ }^{206} \mathrm{~Pb} /{ }^{207} \mathrm{~Pb}$ $\mathrm{e}^{208} \mathrm{~Pb} /{ }^{206} \mathrm{~Pb}$ para os solos da Baía do Sancho são: 1,229 1,234 e 2,035 - 2,041; Praia do Leão: 1,239 - 1,241 e 2,027 - 2,031; Ponta de Atalaia: 1,240 - 1,246 e 2,019 - 2,026 e Mata Ponta Oeste: 1,234 - 1,236 e 2,034 - 2,035, respectivamente. Para uma mesma amostra, as variações entre as composições isotópicas obtidas para a amostra total (T), lixiviado (L) e resíduo (Res) são bem reduzidas, e geralmente as razões mais radiogênicas são medidas nos resíduos e as menos radiogênicas, nos lixiviados. Também, de modo geral, foi observado que as amostras coletadas a $0-10 \mathrm{~cm}$ são ligeiramente menos radiogênicas que aquelas coletadas a $10-20 \mathrm{~cm}$ de profundidade.

No perfil do Morro do Quixaba, o Pb total foi dosado na amostra de superfície $(0-10 \mathrm{~cm})$ e nas amostras de profundidades crescentes até $60-70 \mathrm{~cm}$, e sua concentração mostrou valores decrescentes, de $14,39 \mathrm{ppm}$ a $10,96 \mathrm{ppm}$. As tendências acima esboçadas para o comportamento isotópico do $\mathrm{Pb}$ nos outros perfis de solo estudados são mais nítidas no perfil do Morro do Quixaba: as razões isotópicas dos lixiviados são bem menos radiogênicas $\left({ }^{206} \mathrm{~Pb} /{ }^{207} \mathrm{~Pb}=1,220\right.$ a 1,233) que as dos resíduos $\left({ }^{206} \mathrm{~Pb} /{ }^{207} \mathrm{~Pb}=1,234\right.$ a 1,238), e os resultados da "dissolução total" estão entre os valores 

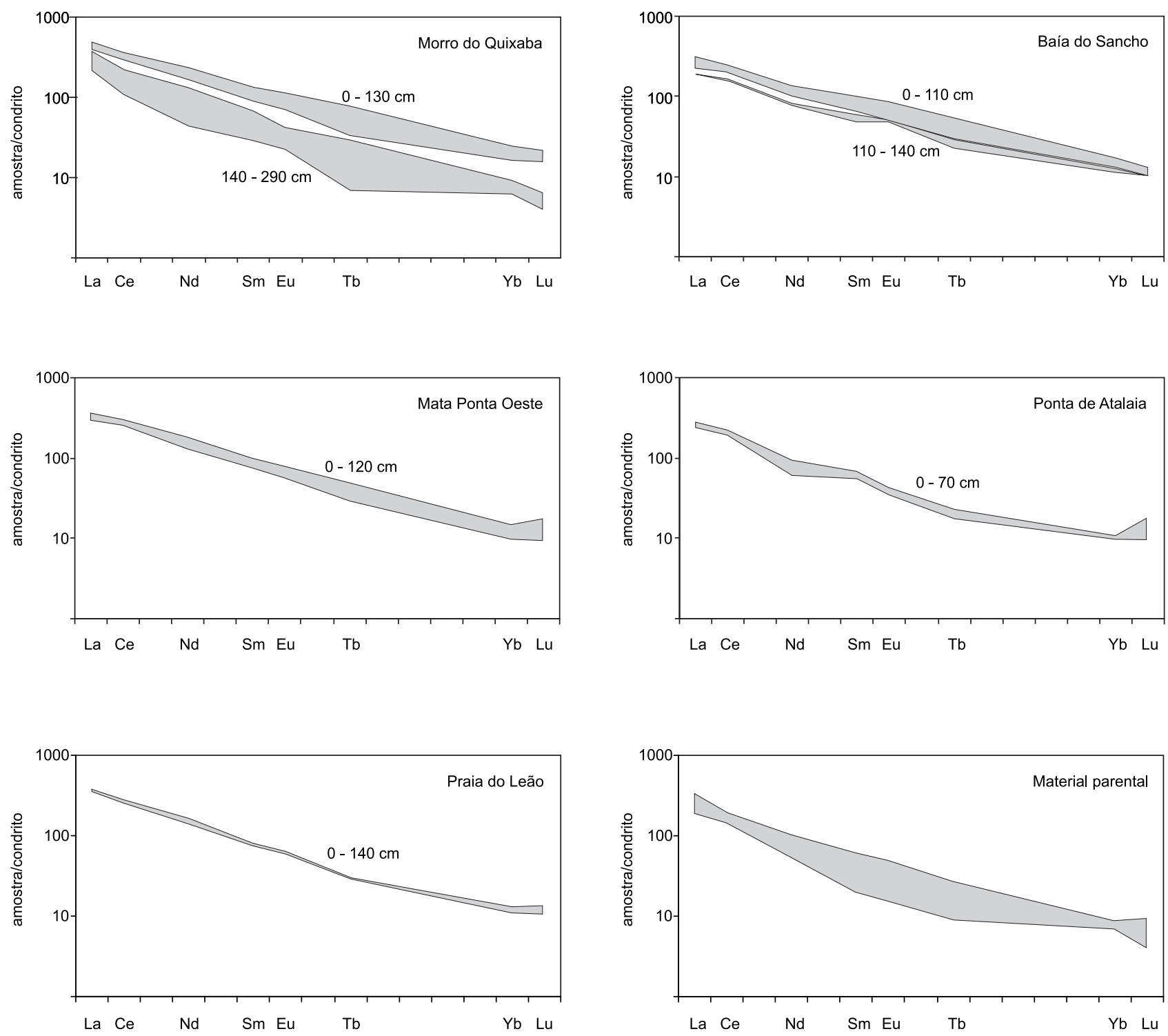

Figura 2. Padrões de ETR dos solos da ilha de Fernando de Noronha e das rochas da Formação Q uixaba e da Formação Remédios (Lopes, 2002; Weaver, 1990), normalizados pelo condrito.

dos lixiviados e dos resíduos. As razões isotópicas de $\mathrm{Pb}$ de todas as amostras do Morro do Quixaba tornam-se sistematicamente mais radiogênicas com a profundidade e os dados dos resíduos são compatíveis com os dados determinados para os nefelina basaltos $\left({ }^{206} \mathrm{~Pb} /{ }^{207} \mathrm{~Pb}=1,242\right.$; Gerlach, Stormer e Mueller, 1987), confirmando a origem geogênica desse chumbo. As razões menos radiogênicas de ${ }^{206} \mathrm{~Pb} /{ }^{204} \mathrm{~Pb}$ $\mathrm{e}^{206} \mathrm{~Pb} /{ }^{207} \mathrm{~Pb}$ observadas nos lixiviados sugerem fortemente a presença de um componente de chumbo de origem antrópica. As razões isotópicas ${ }^{206} \mathrm{~Pb} /{ }^{204} \mathrm{~Pb}$ e ${ }^{206} \mathrm{~Pb} /{ }^{207} \mathrm{~Pb}$ nas amostras lixiviadas crescem linearmente com a profundidade (Figura 3), indicando a influência crescente do componente geogênico nas amostras mais distantes da superfície. A exemplo de casos semelhantes de comportamento dos isótopos de $\mathrm{Pb}$ nos solos, descritos na literatura (Bindler et al., 1999; Semlali et al., 2001), em Fernando de Noronha $\mathrm{o} \mathrm{Pb}$ foi provavelmente adicionado ao solo por meio de deposição atmosférica difusa. Os valores decrescentes dos teores de $\mathrm{Pb}$ total com a profundidade constituem mais uma evidência desse processo. 
Tabela 2. Dados isotópicos de Pb para os solos da ilha de Fernando de $N$ oronha ( $T=$ dissolução total; $L=$ fração lixiviada; Res = resíduo .

\begin{tabular}{|c|c|c|c|c|c|c|c|}
\hline & $206 / 204 \mathrm{~Pb}$ & $\begin{array}{l}\text { Erro } \\
\pm 1 \mathrm{~s} \\
\end{array}$ & $206 / 207 \mathrm{~Pb}$ & $\begin{array}{l}\text { Erro } \\
\pm 1 \mathrm{~s} \\
\end{array}$ & $208 / 206 \mathrm{~Pb}$ & $\begin{array}{l}\text { Erro } \\
\pm 1 \mathrm{~s} \\
\end{array}$ & $\begin{array}{l}\text { Pb total } \\
\text { ppm }\end{array}$ \\
\hline \multicolumn{8}{|c|}{ Baía do Sancho $(\mathrm{cm})$} \\
\hline $0-10 \mathrm{~T}$ & 19,224 & 0,015 & 1,231 & 0,002 & 2,037 & 0,002 & N.D. \\
\hline $10-20 \mathrm{~T}$ & 19,259 & 0,006 & 1,234 & 0,002 & 2,035 & 0,001 & 5,91 \\
\hline $0-10 L$ & 19,215 & 0,013 & 1,229 & 0,002 & 2,041 & 0,003 & N.D. \\
\hline $10-20 \mathrm{~L}$ & 19,223 & 0,032 & 1,233 & 0,009 & 2,035 & 0,003 & N.D. \\
\hline $0-10 \operatorname{Res}$ & 19,240 & 0,005 & 1,231 & 0,001 & 2,040 & 0,002 & N.D. \\
\hline $10-20 \operatorname{Res}$ & 19,244 & 0,003 & 1,233 & 0,002 & 2,035 & 0,002 & N.D. \\
\hline \multicolumn{8}{|l|}{ Praia do Leão $(\mathrm{cm})$} \\
\hline $0-10 \mathrm{~T}$ & 19,373 & 0,022 & 1,240 & 0,003 & 2,029 & 0,002 & N.D. \\
\hline $10-20 \mathrm{~T}$ & 19,381 & 0,008 & 1,240 & 0,002 & 2,028 & 0,001 & 10,591 \\
\hline $0-10 \mathrm{~L}$ & 19,355 & 0,014 & 1,239 & 0,002 & 2,031 & 0,003 & N.D. \\
\hline $10-20 \mathrm{~L}$ & 19,374 & 0,004 & 1,239 & 0,002 & 2,030 & 0,001 & N.D. \\
\hline $0-10 \operatorname{Res}$ & 19,396 & 0,005 & 1,241 & 0,002 & 2,029 & 0,002 & N.D. \\
\hline $10-20 \operatorname{Res}$ & 19,391 & 0,008 & 1,241 & 0,001 & 2,027 & 0,002 & N.D. \\
\hline \multicolumn{8}{|c|}{ Ponta de Atalaia $(\mathrm{cm})$} \\
\hline $0-10 \mathrm{~T}$ & 19,422 & 0,040 & 1,244 & 0,006 & 2,024 & 0,007 & N.D. \\
\hline $10-20 \mathrm{~T}$ & 19,414 & 0,009 & 1,242 & 0,003 & 2,023 & 0,001 & 11,357 \\
\hline $0-10 L$ & 19,421 & 0,011 & 1,242 & 0,002 & 2,026 & 0,003 & N.D. \\
\hline $10-20 \mathrm{~L}$ & 19,389 & 0,004 & 1,240 & 0,001 & 2,026 & 0,001 & N.D. \\
\hline $0-10 \operatorname{Res}$ & 19,465 & 0,007 & 1,244 & 0,001 & 2,024 & 0,001 & N.D. \\
\hline $10-20 \operatorname{Res}$ & 19,468 & 0,012 & 1,246 & 0,002 & 2,019 & 0,002 & N.D. \\
\hline \multicolumn{8}{|c|}{ Mata Ponta Oeste $(\mathrm{cm})$} \\
\hline $0-10 \mathrm{~T}$ & 19,298 & 0,016 & 1,235 & 0,003 & 2,035 & 0,002 & N.D. \\
\hline $10-20 \mathrm{~T}$ & 19,300 & 0,011 & 1,235 & 0,002 & 2,034 & 0,001 & 13,501 \\
\hline $0-10 L$ & 19,302 & 0,008 & 1,234 & 0,002 & 2,035 & 0,003 & N.D. \\
\hline $10-20 \mathrm{~L}$ & 19,283 & 0,004 & 1,234 & 0,001 & 2,035 & 0,001 & N.D. \\
\hline $0-10 \operatorname{Res}$ & 19,313 & 0,004 & 1,236 & 0,001 & 2,034 & 0,001 & N.D. \\
\hline $10-20 \operatorname{Res}$ & 19,311 & 0,005 & 1,234 & 0,001 & 2,035 & 0,001 & N.D. \\
\hline \multicolumn{8}{|c|}{ Morro do Quixaba $(\mathrm{cm})$} \\
\hline $0-10 \mathrm{~T}$ & 19,236 & 0,011 & 1,229 & 0,002 & 2,044 & 0,002 & 14,393 \\
\hline $20-30 \mathrm{~T}$ & 19,275 & 0,016 & 1,231 & 0,003 & 2,041 & 0,005 & 14,185 \\
\hline $40-50 \mathrm{~T}$ & 19,324 & 0,009 & 1,236 & 0,001 & 2,036 & 0,003 & 12,437 \\
\hline $60-70 \mathrm{~T}$ & 19,327 & 0,030 & 1,236 & 0,006 & 2,036 & 0,005 & 10,958 \\
\hline $0-10 L$ & 19,080 & 0,005 & 1,220 & 0,001 & 2,049 & 0,001 & N.D. \\
\hline $20-30 L$ & 19,192 & 0,011 & 1,225 & 0,004 & 2,046 & 0,001 & N.D. \\
\hline $40-50 \mathrm{~L}$ & 19,289 & 0,005 & 1,230 & 0,001 & 2,044 & 0,001 & N.D. \\
\hline $60-70 \mathrm{~L}$ & 19,321 & 0,005 & 1,233 & 0,001 & 2,040 & 0,001 & N.D. \\
\hline $0-10 \operatorname{Res}$ & 19,322 & 0,004 & 1,235 & 0,001 & 2,036 & 0,001 & N.D. \\
\hline $20-30 \operatorname{Res}$ & 19,307 & 0,006 & 1,234 & 0,003 & 2,038 & 0,002 & N.D. \\
\hline $40-50 \operatorname{Res}$ & 19,366 & 0,028 & 1,235 & 0,008 & 2,039 & 0,004 & N.D. \\
\hline $60-70 \operatorname{Res}$ & 19,367 & 0,005 & 1,238 & 0,001 & 2,035 & 0,001 & N.D. \\
\hline
\end{tabular}




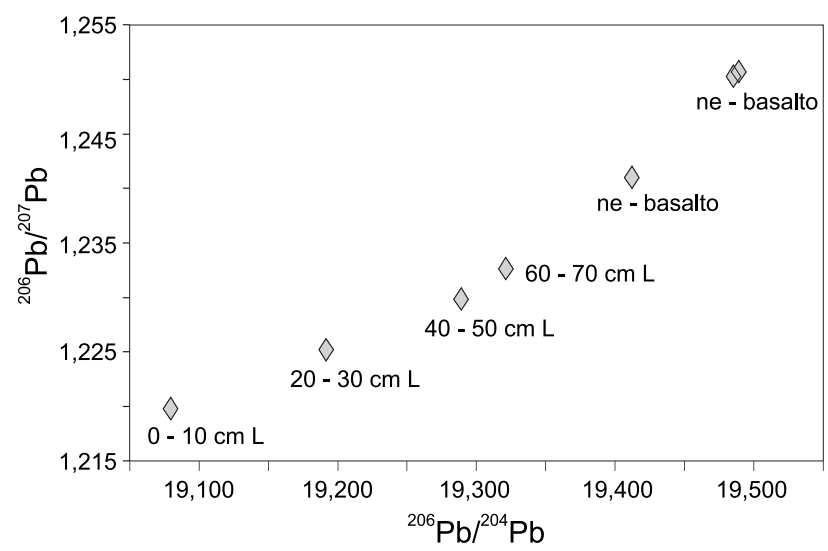

Figura 3. Diagrama ${ }^{206} \mathrm{~Pb} /{ }^{204} \mathrm{~Pb}$ vs. ${ }^{206} \mathrm{~Pb} /{ }^{207} \mathrm{~Pb}$ mostrando a composição isotópica do $\mathrm{Pb}$ da fração lixiviada dos solos do Morro do Q uixaba e dos nefelina-basaltos (Lopes, 2002; G erlach, Stormer, Mueller, 1987).

Estudos anteriores levados a efeito numa lagoa situada na mesma ilha de Fernando de Noronha (Oliveira et al., 2009a) indicaram que os sedimentos da superfície encontram-se contaminados por chumbo de origem antrópica $\left({ }^{206} \mathrm{~Pb} / 207 \mathrm{~Pb}=1,180\right)$, derivado predominantemente de transporte atmosférico de longa distância. A planta aquática $P$. Stratiotes que cobre o lago também apresenta assinatura isotópica não radiogênica $\left({ }^{206} \mathrm{~Pb} /{ }^{207} \mathrm{~Pb}=1,170-1,180\right)$, indicando que registra igualmente a contaminação por $\mathrm{Pb}$ na lagoa.

Em estudos realizados em solos derivados de guano em outra ilha do arquipélago (Ilha Rata), assinatura isotópica de chumbo não radiogênico foi também detectada $\left({ }^{206} \mathrm{~Pb} /{ }^{207} \mathrm{~Pb}=1,171-1,173\right)$ (Oliveira et al., 2009b). Por outro lado, estudos que investigaram o comportamento de metais nos mesmos perfis de solo da ilha de Fernando de Noronha (Oliveira et al., submetido) indicaram a provável contribuição de $\mathrm{Hg}$ aerotransportado nos horizontes mais superficiais do perfil MQ.

Esse conjunto de dados sobre a distribuição de metais em diferentes ambientes do arquipélago de Fernando de Noronha põe em evidência a importância dos processos de contaminação de solos e sedimentos por poluentes aerotransportados, mesmo em ambientes preservados da contaminação direta de fontes poluidoras urbanas ou industriais.

\section{CONCLUSÕES}

Todos os solos analisados, provenientes da alteração de diferentes tipos de rochas vulcânicas aflorantes na ilha de Fernando de Noronha, mostraram-se enriquecidos em ETR em relação às rochas parentais. Dentre os perfis estudados, o do Morro do Quixaba, situado no planalto central da ilha, a $170 \mathrm{~m}$ de altitude, foi o que melhor se prestou, tanto ao estudo mais detalhado do comportamento dos ETR durante a alteração intempérica, quanto ao estudo do comportamento das razões isotópicas de $\mathrm{Pb}$ a partir da superfície. Atingindo $290 \mathrm{~cm}$ de profundidade de amostragem, o perfil MQ abrangeu um amplo intervalo de variação da razão $\mathrm{Si} / \mathrm{Al}$ (1,2 na base a 0,8 no topo), o que permitiu acompanhar de perto a variação dos ETR e seu possível fracionamento com a intensidade do processo intempérico, do qual a referida razão é um índice. Nesse perfil, ficou evidente a tendência geral de concentração dos ETR com a intensificação do intemperismo, o que vem acompanhado de fracionamento, por meio do qual se concentram preferencialmente os ETRP em relação aos ETRL.

Os dados isotópicos do $\mathrm{Pb}$ mostraram expressiva contribuição de $\mathrm{Pb}$ antrópico nos níveis mais superficiais dos solos estudados, sobretudo no perfil do Morro do Quixaba. Isso foi revelado por uma assinatura isotópica do $\mathrm{Pb}$ menos radiogênica nas frações lixiviadas em relação às amostras totais, e nas amostras de superfície, em relação às amostras de profundidade. A assinatura isotópica do $\mathrm{Pb}$ encontrado na fração lixiviada das amostras de solo da superfície $\left({ }^{206} \mathrm{~Pb} /{ }^{207} \mathrm{~Pb}=1,220\right)$ afasta-se dos valores geogênicos típicos das rochas do arquipélago $\left({ }^{206} \mathrm{~Pb} /{ }^{207} \mathrm{~Pb}=1,242\right)$, tendendo aos valores mais característicos do $\mathrm{Pb}$ antrópico derivado da queima da gasolina $\left({ }^{206} \mathrm{~Pb} /{ }^{207} \mathrm{~Pb}=1,192\right.$ : Gioia et al., 2006) e das emissões industriais do hemisfério sul $\left({ }^{206} \mathrm{~Pb} /{ }^{207} \mathrm{~Pb}=1,184\right.$ : Bollhöffer e Rossman, 2000).

\section{AGRADECIMENTOS}

Os autores agradecem particularmente ao Dr. René Boulet e à Dra. Heloisa Filizola pela assistência na amostragem, e são reconhecidos ao Dr. A. Siffedine e ao Prof. E. Menor pela participação no trabalho de campo. São igualmente gratos aos dois revisores anônimos que contribuíram para o aperfeiçoamento do texto final. FAPESP e CNPq forneceram apoio financeiro ao projeto.

\section{REFERÊNCIAS}

ALMEIDA, F. F. M. DE. Geologia e petrologia do Arquipélago de Fernando de Noronha. Rio de Janeiro: Departamento Nacional da Produção Mineral - DNPM, 1955. p. 1-181. (Monografia, n. 13).

BINDLER, R.; BRANNVALL, M. L.; RENBERG, I.; EMTERYD, O.; GRIP, H. Natural lead concentrations. Environmental Science and Technology, v. 33, p. 3362-3367, 1999. 
BOLLHÖFFER, A.; ROSMAN, K. J. R. Isotopic source signatures for atmospheric lead: the Southern Hemisphere. Geochimica et Cosmochimica Acta v. 64, n. 19, p. 3251-3262, 2000.

CASPARI, T.; BAUMLER, R.; NORBU, C.; TSHERING, K.; BAILLIE, I. Geochemical investigation of soils developed in different lithologies in Buthan, Easthern Himalayas. Geoderma, v. 136, n. 1-2, p. 436-458, 2006.

GERLACH, D. C.; STORMER, J. C.; MUELLER, P. A. Isotopic geochemistry of Fernando de Noronha. Earth and Planetary Science Letters, v. 85, n. 1-3, p. 129-144, 1987.

GIOIA, S. M. C. L. Caracterização da assinatura isotópica do chumbo atual na atmosfera e no sistema lacustre do Distrito Federal e pré-antropogênica em Lagoa Feia (GO). 2004. Tese (Doutorado) - Instituto de Geociências, Universidade de Brasília, Brasília, 2004.

GIOIA, S. M. C. L.; PIMENTEL, M. M.; TESSLER, M.; DANTAS, E. L.; CAMPOS, J. E. G.; GUIMARÃES, E. M.; MARUOKA, M. T. S.; NASCIMENTO, E. L. C. Sources of anthropogenic lead in sediments from an artificial lake in Brasilia, central Brazil. The Science of the Total Environment, v. 356, p. 125-142, 2006.

LOPES, R. P. O vulcanismo do arquipélago de Fernando de Noronha, PE: química mineral e geoquímica. 2002. Tese (Doutorado) - Instituto de Geociências, Universidade de São Paulo, 2002.

MARQUES, F. A. Caracterização e classificação de solos da ilha de Fernando de Noronha (PE). 2004. $101 \mathrm{f}$. Dissertação (Mestrado) - Universidade Federal Rural de Pernambuco, Recife, 2004.

MIRARIK, L.; ZIGOVA, A.; BENDL, J.; SKRIVAN, P.; ST'ASTNY, M. The behavior of rare-earth elements and $\mathrm{Y}$ during rock weathering and soil formation in the Ricany granite massif, Central Bohemia. The Science of Total Environment, v. 215, p. 101-111, 1998.

OLIVEIRA, S. M. B.D E.; IMBERNON, R. A. L. Weathering alteration and related REE concentration in the Catalão I carbonatite complex, central Brazil. Journal of South American Earth Sciences, v. 11, n. 4, p. 379-388, 1998.

OLIVEIRA, S. M. B. de; PESSENDA, L. C. R.; GOUVEIA, S. E. M.; BABINSKI, M.; FAVARO, D. I. T. A geochemical and lead isotopical record from a small pond in a remote equatorial island, Fernando de Noronha, Brazil. Holocene, v. 19, n. 3, p. 433-442, 2009a.

OLIVEIRA, S. M. B. de; PESSENDA, L. C. R.; GOUVEIA, S. E. M.; FAVARO, D. I. T. Evidência geoquímica de solos formados pela interação de guanos com rochas vulcânicas, Ilha Rata, Fernando de Noronha (PE). Revista do Instituto de Geociências, v. 9, n. 3, p. 3-12, $2009 \mathrm{~b}$.

OLIVEIRA, S. M. B. de; PESSENDA, L. C. R.; GOUVEIA, S. E. M.; FAVARO, D. I. T. Heavy metal concentrations in soils from a remote oceanic island, Fernando de Noronha, Brazil. Anais da Academia Brasileira de Ciências (submetido).

PATINO, L. C.; VELBEL, M. A.; PRICE, J. R.; WADE, J. A. Trace element mobility during spheroidal weathering of basalts and andesites in Hawaii and Guatemala. Chemical Geology, v. 202, n. 3-4, p. 343-364, 2003.

SHARMA, A.; RAJAMANI, V. Major element, REE, and other trace element behavior in amphibolite weathering under semiarid conditions in Southern India. The Journal of Geology, v. 108, n. 4, p. 487-496, 2000.

SEMLALI, R. M.; OORT, F. V.; DENAIX, L.; LOUBET, M. Estimating distributions of endogenous and exogenous $\mathrm{Pb}$ in soils by using $\mathrm{Pb}$ isotopic ratios. Environmental Science and Technology, v. 35, p. 4180-4188, 2001.

WEAVER, B. L. Geochemistry of highly-undersaturated ocean island basalt suites from the South Atlantic Ocean: Fernando de Noronha and Trindade islands. Contributions to Mineralogy and Geology, v. 105, n. 5, p. 502-515, 1990.

WEDEPOHL, K. H. The composition of the continental crust. Geochimica et Cosmochimica Acta, v. 59, n. 7, p. 1217-1232, 1995.

WEIJDEN, C. H. van der; WEIJDEN, R. D. van der. Mobility of major, minor and some redox-sensitive trace elements and rare-earth elements during weathering of four granitoids in central Portugal. Chemical Geology, v. 125, n. 3-4, p. 149-167, 1995.

WOODHEAD, J. D.; HERGT, J. M. Pb-isotope analyses of USGS Reference Material. Geostandards Newsletter, v. 24, n. 1, p. 33-38, 2000. 